\title{
Stem cells and models of astrocytomas
}

\author{
Deepak Kamnasaran, $\mathbf{P h D}^{1,2}$
}

${ }^{1}$ Department of Pediatrics, Laval University
${ }^{2}$ Pediatrics Research Unit, Centre de recherché du
CHUL

Manuscript submitted 25th December, 2008

Manuscript accepted 10th March, 2009

Clin Invest Med 2009; 32 (2): E166-E179.

\begin{abstract}
Purpose: To provide a critical assessment of current stemcell based pre-clinical models of astrocytomas (gliomas).

Methods: Data were archived from MEDLINE using Boolean formatted keyword queries. Top articles were selected for critical analyses depending on the qualitative assessment of the citation index, novelty of the findings, reputation of the research group and relevance to stem-cell based pre-clinical models of astrocytomas.

Results: The emergence of stem-cell based pre-clinical models of gliomas offers advantages for cellular transformation studies over other current in-vitro cell cultured based models. Cells utilized in these stem-cell based preclinical models are easier to transform, with the induced tumours demonstrating very high molecular and pathological recapitulations of astrocytomas that are derived from humans. These stem-cell based models fall into two categories. In the first, synthetic astrocytes can be differentiated from various stem cell sources such as the nervous system and embryos, and utilized in elegant forward genetic strategies to develop novel astrocytoma pre-clinical models. The second category represents a cancer stem cell pre-clinical model. In this model, glioma stem cells exhibit very high pathological recapitulations of the human tumours, and can be very informative to comprehend the basis of radio-chemoresistance among patients.

Conclusion: The quest to develop robust pre-clinical models of astrocytomas is on an ongoing basis. The models are of clinical importance for the discovery of effective treatment modalities that can considerably improve the health of patients with this deadly disease.
\end{abstract}

Primary brain tumours are among the top 5 causes of cancer-related deaths in the adult and pediatric populations ${ }^{1}$, with gliomas accounting for the majority of cases $^{2}$. Astrocytomas are the most common sub-type of glioma, with four pathological grades as classified by the World Health Organization (WHO) (Figure 1) $)^{3}$. Glioblastoma multiforme (GBM) is the most common malignant astrocytoma, which despite current therapy (surgery, radiation and chemotherapy), has a median survival of about 1 year. ${ }^{4}$ Although pathologically indistinguishable, there are two subtypes of GBMs. Primary GBMs $(60 \%$ of subjects $>50 \mathrm{yr})$ arise rapidly or de novo without any previous clinical or histopathological history, whereas secondary GBMs (40\% of subjects $<45 \mathrm{yr}$ ) derive from preexisting low-grade lesions after long latency ranging from 5 to $10 \mathrm{yr}$ (Kleihues and Cavenee, 2000), both of which correspond to at least two genetic progression paradigms (Figure 2). 3,5,6 These sub-types of GBMs also have contrasting responses to current therapies, most likely as a consequence of differences in the genetic pathogenesis.

Overall, astrocytomas like other tumours are molecularly heterogeneous with numerous genetic alterations, including single gene defects and chromosome aberrations that are of epidemiological, prognostic and therapeutic relevance. Common to all astrocy- 


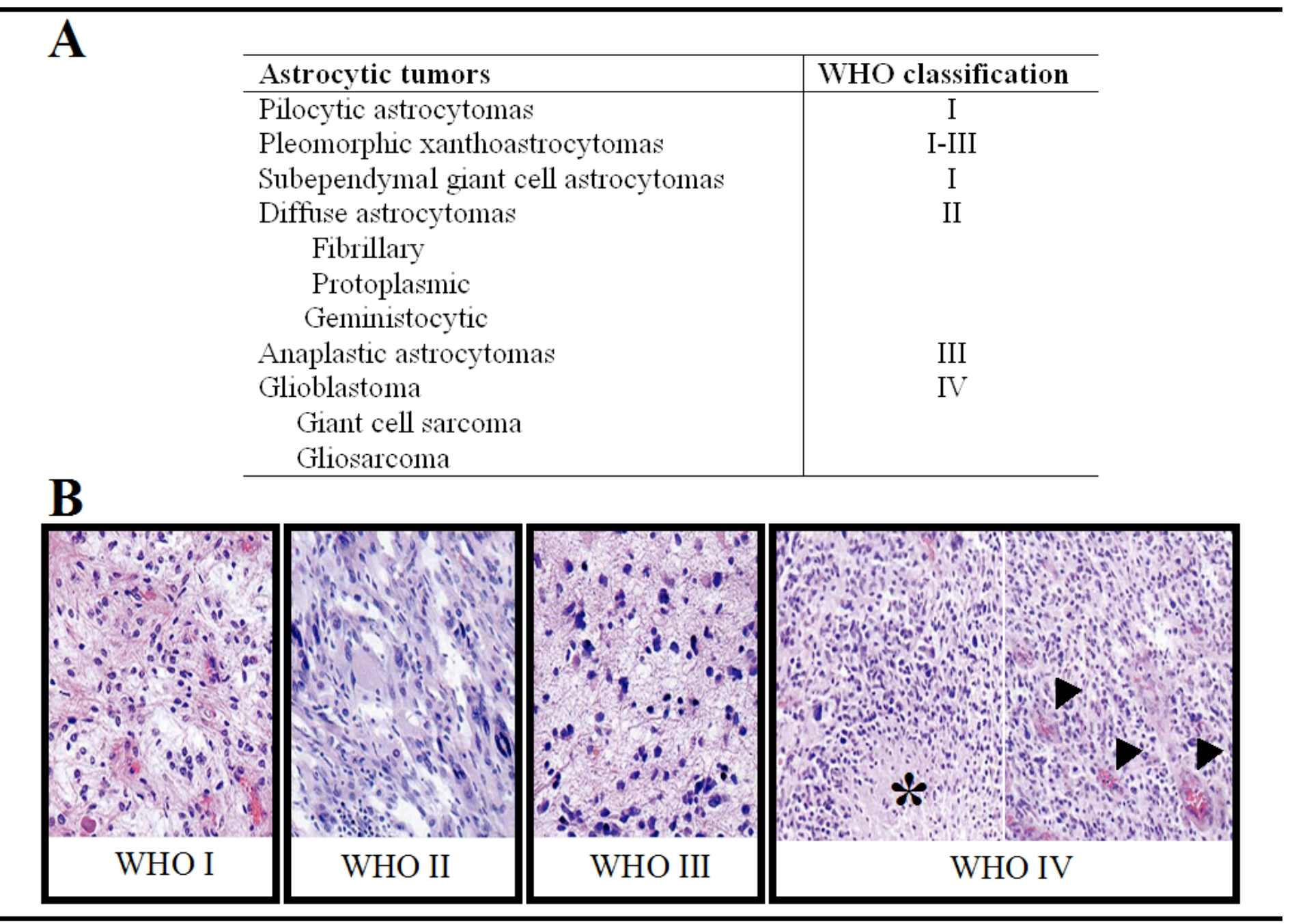

FIGURE 1. Classifications of astrocytic tumors according to the World Health Organization (WHO). (A) Pathological variations of astrocytomas within each gradations. (B) Pathological examples of each gradations of astrocytomas. Grade I astrocytomas are usually indolent with limited invasive potentiation. Some tumors may demonstrate microvascular pleomorphism and cellular pleomorphism. Grade II astrocytomas have cellular pleomorphism and limited invasiveness. Grade III astrocytomas have a profound increase in mitotic index, moderate degree of cellular pleomorphism and nuclear atypia. Grade IV astrocytomas demonstrate pathological features of Grade III astrocytomas in conjunction with necrotic sites (asterisks) and microvascular hyperplasia (arrows). Of note, sample pictures of each gradation of astrocytomas are taken from N Engl J Med 2005 353(8):811-22, with permission.

tomas are aberrations in cell-cycle regulation, mediated by both $\mathrm{p} 53$ and $\mathrm{pRb}$ regulated pathways, plus several aberrant growth factor-receptor-downstream signaling pathways. ${ }^{7}$ As part of the ongoing Cancer Genome Project and numerous independent international groups, our understanding of the genetic basis of astrocytomas has been significantly enlightened. ${ }^{9-14}$ For instance, over 220 regions of the human genome are discovered with a gain or loss of genetic content in patients with primary or secondary glioblastoma multiforme ${ }^{14}$.

In an effort to eradicate this deadly disease among patients, there is a continual trend towards the development of robust pre-clinical models of astrocytomas. An ideal pre-clinical model must conservatively recapitulate both the pathological and molecular properties inherent in the patients' tumours. The model must also be amenable towards therapeutic response studies in 
Secondary

Glioblastoma

\section{Glioma Precursor Cell}

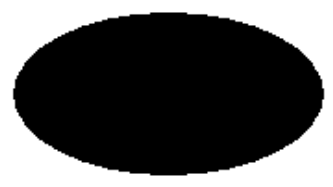

PDGFRa

PDGFa

p53

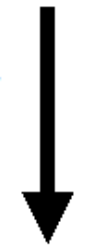

Low grade astrocytoma (WHO grade II)

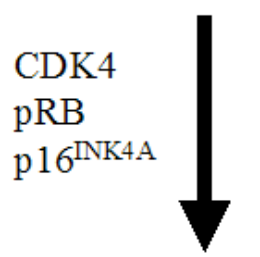

Anaplastic astrocytoma (WHO grade III)

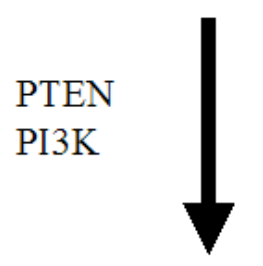

\section{Primary \\ Glioblastoma}

p16 $6^{\mathrm{INK} 4 \mathrm{~A}}$

PTEN

CDK4

EGFR

VEGF

p14 ARF

$\mathrm{PDGFb}$

PDGFRb

VEGF

VEGFR

TIE

ANG

\section{Glioblastoma multiforme (WHO grade IV)}

FIGURE 2. The genetic pathogenesis of glioblastoma multiforme. Primary glioblastoma arises from a direct transformation of a glioma precursor cell which typically involves concurrent mutations in several genes such as EGFR, VEGFR, and so on. Secondary glioblastoma arises from a progressive series of pathological events with each progression step associated with a distinct cocktail of genetic mutations. Of note, both types of glioblastomas are associated with shared and with distinct genetic signatures.

our effort to discover treatments with outstanding efficacies. Recent discoveries have prompted more of a focus on the stem cell, progenitor cell and earlydifferentiated cell type lineages in the origin of astrocytomas, which is of therapeutic relevance. This article describes some of the current efforts that are established to develop novel pre-clinical models of astrocytomas, by specifically addressing whether these early lineage cell types can be implemented in the development and therapeutic applications of robust models. At this time, it is important for physicians to become acquainted with the prospects of these novel stem-cell based pre-clinical models, which are anticipated to revolutionize the way of how we understand the biology of astrocytomas. This body of knowledge will inevitably be of clinical importance in devising effective therapeutic modalities, as an advancement towards the betterment in the treatment and care of these terminally ill patients. 


\section{Methods}

Data were archived from MEDLINE, using Boolean formatted queries on the keywords including: glioma stem cells, stem cells, astrocytoma, glioblastoma multiforme, astrocytes, synthetic astrocytes, progenitor cells, ENU, MNU, viral, brain tumours and brain tumour model. Only the top articles were selected for critical analyses depending on the qualitative assessment of the citation index, novelty of the findings, reputation of the research group and relevance to stem-cell based pre-clinical models of astrocytomas.

\section{Results}

\section{Current perspectives on the origin of astrocytomas}

An understanding of the origin and progression of astrocytomas is pertinent towards the eradication of this incurable disease. In fact, both the assortment of specific genetic mutations and cell types contribute towards the origin of gliomas. Mature astrocytes can dedifferentiate and transform into astrocytomas depending on the type of mutation. For instance, an increase in the expression of the Platelet Derived Growth Factor Receptor-Beta gene can de-differ-entiate and transform mature astrocytes. ${ }^{15}$ However, the major pitfall of this postulate is the inability to explain the origin of tumours with mixed ontogenies.

An alternative hypothesis on the origin of astrocytomas is based on the neoplastic transformation of early cell lineage types that have a stem-cell like property. ${ }^{16}$ It is not precisely defined what specific cell types contribute towards the stem-cell origin of gliomas. However, Neural stem cells (NSC), glial progenitor cells and early-differentiated/immature glial cells are speculated as being the most likely candidates. Niches for these cell types are in the subventricular zone (SVZ), dentate gyrus, hippocampus and subcortical white matter ${ }^{16}$. In fact, astrocytes in the SVZ demonstrate stem-cell like properties ${ }^{17}$. These cells share common features such as an enhanced proliferation, high motility, multi-potency, as- sociation with blood vessels, regulation by many cellular pathways that are evident in brain tumours, and the expression of immature antigenic phenotypes ${ }^{16}$.

\section{Animal model studies on the origin of astrocytomas}

\section{Early evidence}

Many decades ago, preliminary evidence was provided to strengthen the postulate that early cell lineages are implicated in the origin of brain tumours including findings of the sub-ventricular zone as being more sensitive to chemical and viral oncogenesis in comparison to other central nervous system areas harboring a low proportion of proliferating cells. ${ }^{18-20}$ For instance, in canine and rodent brains, avian sarcoma viral transformation or systemic exposure to the $\mathrm{N}$ ethyl-N-nitrosourea chemical mutagen preferentially succumb to tumor development in the sub-ventricular zone. ${ }^{19,20}$ These preliminary experiments however were uninformative in dissecting the genetic etiology of brain tumours.

\section{Somatic transgenesis evidence}

Mouse models of gliomas have been developed such that glioma potentiation can be induced using somatic transgenesis methods. With somatic transgenesis, cocktails of interacting known genes carried in engineered lentiviruses, retroviruses or adenoviruses can be stereotactically delivered to specific regions of the rodent brain to transduce certain cell types. This method has been successful in demonstrating that mutating the neural stem cells, neuro-progenitor cells and early differentiated astrocytes within or juxtaposed to the sub-ventricular zone with elevated expression of oncogenes such as EGFR, AKT and RAS, can induce the development of high grade astrocytomas. ${ }^{21-24}$ This is in contrast to the findings of murine mature astrocytes from the outer cortex that are less susceptible to transform when mutant. ${ }^{21-24}$ Although this method is very powerful to understand the origin of astrocytomas, the use of lentiviruses and retroviruses can 
sometimes mutate endogenous genes of the transduced cells that can potentially affect transformation. Furthermore, adenoviruses are only biased towards transducing cells that only express the CAR receptors. ${ }^{25}$

\section{Germline transgenesis evidence}

Germline transgenesis has been effectively utilized to develop an assortment of Genetically Engineered Mouse (GEM) models of gliomas. However, the pitfall of these mouse models stems from the observation of frequent variable penetrance for tumour development. Despite this, these mouse models have an inherent developmental microenvironment to facilitate an understanding of how specific genes interact, and how stromal cells interact with tumour cells during astrocytomagenesis. For instance, mice with ubiquitous loss of function mutations in the p53 tumour suppressor gene in conjunction with a loss of function mutation in the NF1 tumour suppressor gene only in astroglial cells, can succumb to developing glioblastoma multiforme within 4 months after birth. These tumours originate from the sub-ventricular zone of the cerebrum and express multi-lineage cellular markers, including that of stem cells. ${ }^{26}$

TABLE 1: Pros and Cons of non-stem cell based cell culture models of astrocytomas

\begin{tabular}{l|l|l}
\hline $\begin{array}{l}\text { Somatic } \\
\text { astrocyte } \\
\text { cultures }\end{array}$ & $\begin{array}{l}\text { Preserves cellular plus } \\
\text { genetic architectures } \\
\text { Can be useful for } \\
\text { forward genetic studies }\end{array}$ & $\begin{array}{l}\text { Pros } \\
\text { Difficult to establish and } \\
\text { propagate } \\
\text { Senescence is inevitable } \\
\text { Not very conducive for } \\
\text { transformation studies }\end{array}$ \\
\hline $\begin{array}{l}\text { Glioma cell } \\
\text { lines }\end{array}$ & $\begin{array}{l}\text { Easy to propagate } \\
\text { Preserves some genetic } \\
\text { plus pathological } \\
\text { signatures of the host } \\
\text { tumor } \\
\text { Preserves some } \\
\text { therapeutic response } \\
\text { signatures of the host } \\
\text { tumour }\end{array}$ & $\begin{array}{l}\text { De-novo cellular, } \\
\text { molecular and } \\
\text { pathological signatures } \\
\text { Cellular heterogeneity can } \\
\text { arise } \\
\text { Complex genetic } \\
\text { signatures }\end{array}$ \\
\hline
\end{tabular}

\section{Cell culture based pre-clinical models of astrocytomas}

Somatic Astrocyte cell culture pre-clinical models and pitfalls

As an alternative to the use of glioma cell lines to develop glioma models, somatic astrocytes can be utilized (Table 1). It must be noted that when in-vitro somatic astrocyte cultures are established, the majority of cells that remain in culture are more reminiscent of mature or terminally differentiated astrocytes. To facilitate these mature somatic astrocytes to become conducive to transform, pre-immortalization is pertinent. Pre-immortalization can be achieved either spontaneously or via the constitutive expression of genes such as telomerase to bypass cellular senescence. ${ }^{27-29}$ For instance, when pre-immortalized human mature somatic astrocytes are mutated to express the human papillomavirus 16 E6 and E7 genes in conjunction with the oncogenes RAS and AKT or the GATA6 tumour suppressor gene, de-differentiation and transformation into high grade astrocytomas occurs. $^{27-29}$

The use of somatic astrocytes however has several pitfalls to develop models of gliomas (Table 1). These include difficulty in the establishment and in-vitro propagation while in culture. For instance, human mature astrocytes have a finite lifespan in culture after 20 days upon which entry is into cellular senescence. ${ }^{27}$ Another pitfall is that there is a paucity of somatic astrocytes that are non-transformed but have mutant genetic backgrounds. Transformation studies warrant multiple mutations to induce full de-differentiation and transformation. In light of these pitfalls, it is therefore imperative to explore alternative in-vitro strategies in the creation of efficient and robust glioma models.

Clin Invest Med • Vol 32, no 2, April 2009

E170 


\section{Alternative astrocytoma cell cuture based pre- clinical models}

\section{Definition of synthetic cell lineages}

Stem cells and progenitor/precursor cells can be used to differentiate into multi-lineage cells under various cell-culturing conditions. These cells that are differentiated from stem cells or progenitor/precursor cells need to be designated as being synthetic cell lineages. Many investigators have designated these differentiated cells as an astrocyte, neuron and so on. However, to do so is problematic. The term synthetic cell lineage is more appropriate since these cells are synthesized under user-defined in-vitro cell culture conditions and therefore lack the normal developmental and physiological cues that are necessary to develop into somatic astrocytes. Secondly, there is some controversy as to what exactly are these cells that have been differentiated from the stem cell/progenitor/precursor cell sources. For instance, studies on the neurons and astrocytes that are differentiated from skin precursor cells have demonstrated them as being neuron-like or astrocyte-like with the expression of some terminally differentiated markers, however they are in fact non-functional. ${ }^{30,31}$.

\section{Sources of synthetic astrocytes}

Synthetic astrocytes have been differentiated under various cell-culturing conditions from numerous stem cell and progenitor/precursor cell sources. These include umbilical hematopoietic stem cells, bone marrow stem cells, neural stem cells, embryonic stem cells, skin precursor cells and glial restricted precursor cells. ${ }^{30,32-38}$ There are a multitude of strategies utilized to synthesize astrocytes under user-defined in-vitro conditions. These include treatments of the stem/ progenitor/precursor cell sources with retinoic acid ${ }^{39}$, growing cells on a layer of endothelial cells ${ }^{40}$ or astrocytes $^{41}$, growth in the presence of cytokines such as Epidermal growth factor $(\mathrm{EGF})^{42}$, Ciliary neurotrophic factor $(\mathrm{CNTF})^{43}$, Activin ${ }^{44}$, Fibroblast growth factors (FGFs) ${ }^{45}$ and Bone Morphogenetic proteins. ${ }^{46}$ However, the final yield of synthetic astrocytes is very variable. The use of a more stringent and step-wise differentiation process can substantially increase the yield of synthetic astrocytes. ${ }^{33}$

\section{Usefulness of synthetic astrocytes to develop astrocytoma pre-clinical models}

Synthetic astrocytes can be excellent cellular sources in the derivation of robust pre-clinical models of gliomas (Figure 3). They offer the major advantage of having increased genetic and pathological stability in contrary to glioma stem cell pre-clinical models. ${ }^{33}$ Of course, it must be recognized that these in-vitro based pre-clinical models are challenged by the lack of relevant developmental, epigenetic and microenvironment cues reminiscent in the animal preclinical models. Such factors can ultimately influence the genetic, pathological, plus treatment response properties of the induced tumours, and can inevitably challenge the robustness of these in-vitro based preclinical models. However, since animal pre-clinical models are less efficient and costly, the development of alternative models such as stem-cell based preclinical models need to be explored, and can in fact be used to complement the animal pre-clinical models.

\section{Applications of synthetic astrocytes to model} astrocytomas

Although synthetic astrocytes were differentiated from many stem cell and progenitor/precursor cell sources in the past ${ }^{32-38}$, only two sources have been utilized so far in the development of astrocytoma models. These two sources, described below, include both neural stem cells and a non-neural stem cell, namely embryonic stem cells. They have been most useful to comprehend the biology of astrocytomas using elegant forward genetics strategies. 


\section{Genetic alterations of synthetic differentiated cell lineages}

$\checkmark$

Candidate genes

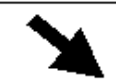

Random mutagenesis

\section{Altered differentiation Properties}

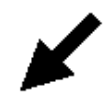

Morphology Proliferation Motility Invasion Transformation
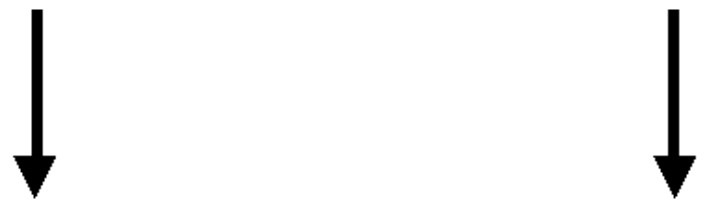

Altered proteome \& transcriptome

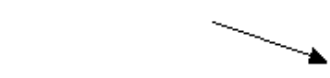

Pathology

Confirmation studies among human brain tumor specimens

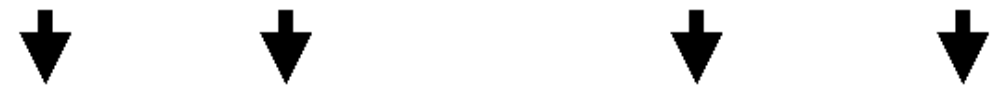

\section{MOLECULAR MECHANISM THERAPEUTIC DESIGN \& TESTING}

FIGURE 3. An outline of the spectrum of applications that can be associated with the use of synthetic cell lineages, such as synthetic astrocytes. These cellular substrates which are more reminiscent in the origin of astrocytomas can inevitably assist in deciphering the molecular mechanisms of astrocytomagenesis, and to serve in elegant therapeutic design and testing studies.

\section{Source - Neural stem cell (NSC) differentiated synthetic astrocytes}

\section{Cellular and transformation properties}

Somatic neural stem cells were initially postulated to be prevalent only in the embryonic/fetal brain. However, postnatal and adult neural stem cells are also evident. ${ }^{47-49}$ Furthermore, synthetic neural stem cells can be differentiated from stem cell sources such as embryonic stem cells and mesenchymal stem cells. ${ }^{50,51}$ In fact, both somatic neural stem cells and synthetic neural stem cells can be differentiated into synthetic astrocytes under various growth factor treatments ${ }^{40-46}$, and with varying efficiency. Most remarkable, in a study of synthetic astrocytes differentiated from murine neural stem cells, these synthetic astrocytes are deemed to be most similar to immature somatic astrocytes, and the ability to self-renew with an enhanced proliferation. ${ }^{32}$ This is in contrarst to mature murine somatic astrocytes that enter cellular senescence by the $10^{\text {th }}$ passage upon culturing. ${ }^{32}$ Neither the synthetic nor somatic murine astrocytes transform in-vitro or in-vivo. However, these synthetic astrocytes when mutated with a cocktail of glioma genes such as 
the p53 tumour suppressor gene and EGFR oncogene, have a higher propensity to transform, than mature murine somatic astrocytes, which do not. In this manner these synthetic astrocytes demonstrate in-vitro anchorage independent growth in culture, and can grow into high grade differentiated gliomas intra-cranially in immuno-deficient mice. ${ }^{32}$

\section{Source - Embryonic stem cell (ESC) differentiated synthetic astrocytes}

\section{Cellular and transformation properties}

Embryonic stem (ES) cells have been proposed for many applications including tissue repair and regeneration, plus embryonic stem cell transgenesis. They can also be differentiated into specific cell types with prospects to understand the process of cell differentiation and gliomagenesis. ES cells were initially established from pluripotent cells that reside in the inner cell mass (epiblast) of murine blastocysts ${ }^{52,53}$ with subsequent isolation from other primate embryos such as the marmoset, cynomolgus and rhesus monkeys, and humans. ${ }^{54-58}$ They are characterized by the ability to self-renew for indefinite periods and clonogenic property. Under locally controlled conditions comprising of an assortment of growth factors or in-vivo micro-environment cues, these ES cells can differentiate into multiple cell types representative of all three germ layers, including synthetic astrocytes, with demonstrative characteristic morphology and specialized functions. ${ }^{59-62}$

Like synthetic astrocytes differentiated from murine neural stem cells, synthetic astrocytes differentiated from embryonic stem cells are most similar to immature somatic astrocytes. ${ }^{33}$ These cells can selfnew with an enhanced proliferation and express immature antigenic phenotypes. While embryonic stem cells exhibit anchorage independent growth and can grow into tumours reminiscent of teratomas ${ }^{33,54,56}$, the synthetic astrocytes do not. ${ }^{33}$ Most remarkable, these synthetic astrocytes have a higher propensity to transform when mutated with a cocktail of glioma onco- genes such as RAS, AKT and MDM2, than murine mature somatic astrocytes, which do not. In this manner, these mutated synthetic astrocytes demonstrate anchorage independent growth and can grow into intra-cranial tumours in immuno-deficient mice, which are predominantly and pathologically similar to undifferentiated high grade gliomas. ${ }^{33}$

\section{Pitfalls of using synthetic astrocyte pre-clinical models}

Overall, these synthetic astrocytes that are most similar to immature somatic astrocytes seem to be more reminiscent in the origin of gliomas, when mutant. ${ }^{32,33}$ These findings transcend prospects for future research to address the robustness of these models. However, it must be recognized that certain mutations in the stem cell or precursor/progenitor cell source can affect differentiation into a synthetic astrocyte cell lineage without succumbing to transformation into a tumour of astrocytic ontogeny. For instance, the overexpression of Bone Morphogenic Protein (BMP) in transgenic murine neural stem cells induces an increase in astroglial lineage commitment ${ }^{63}$, while murine embryonic stem cells that are mutant for both copies of the GP130 gene do not differentiate in-vitro at all into synthetic astrocytes from neuroepithelial progenitor cells. ${ }^{64}$ In a similar manner, embryonic stem cells with losses of both copies of the PTEN, PEM or APC genes induce severely perturbed differentiation capabilities. ${ }^{65-68}$ There are however some mutations such as losses of both copies of the c-FOS gene, or a loss of a single copy of the p53 gene that do not alter the differentiation potential of the stem cell source. ${ }^{33,69}$

\section{Cancer stem cells as a model for gliomas}

The Cancer stem cell hypothesis ${ }^{70}$ has gained momentum within the field of oncology. A cancer stem cell is defined as a cell within a tumour that has the capacity to self-renew and to differentiate into a heterogeneous lineage of cells. ${ }^{70}$ Cancer stem cells have been identi- 
fied from many types of tumours including hematological, colon, breast, bone, skin, lung, ovarian and brain. ${ }^{70}$ Neurosphere-like-forming cells, now known as tumourspheres, were isolated from both pediatric and adult gliomas, and even glioma cell lines. ${ }^{71-79}$ Of most clinical importance, glioma stem cells are suspected to be responsible for tumour recurrence and current treatment resistance among patients. ${ }^{80-83}$.

\section{Cellular properties}

Potential sources of glioma stem cells include mutations in neural stem cells and the persistent progenitor astrogial cells of the sub-ventricular zone. ${ }^{84-86}$ Table 2 summarizes the similarities and differences between a glioma stem cell and a normal neural stem cell. Indeed, a small sub-population of brain tumour stem cells characterized by the expression of the cell surface CD133 protein, and low retention of the Hoechst 33342 dye in the cell nucleus, were isolated from astrocytomas. ${ }^{71-77,87}$ These $\mathrm{CD} 133^{+}$cells are in low abundance with an estimated population of $\sim 0.01-5 \%$ of the tumour mass, unlike the remaining cells of the tumour mass that are terminally differentiated and cell-death committed. However, there is a positive correlation between the abundance of $\mathrm{CD} 133^{+}$cells and the aggressiveness of the astrocytoma. ${ }^{71-75,87}$ These cells form tumourspheres in culture that have about 600-800 adhering cells. The rate of tumoursphere formation is significantly faster compared to neurosphere formation of neural stem cells. ${ }^{72-77}$ Glioma stem cells can self-renew in culture with an enhanced proliferation unlike mature glial cells. ${ }^{72-77}$ This proliferative property may be, in part, due to the increased activity of the telomerase protein ${ }^{88,89}$ that retards the shortening of the telomeres, and subsequently attenuates cellular senescence or even cell death. Glioma stem cells that form tumourspheres faster have a positive correlation with a more aggressive or higher gradation of gliomas. ${ }^{73,75,82}$

Glioma stem cells can also differentiate into multilineage cells under in-vitro cell culture conditions and upon in-vivo growth. ${ }^{71-75}$ However, with the heterogeneous population of multi-lineage cells, there is a bias towards the cells that express the astrocytic marker GFAP, and less often cells that express neuronal markers and oligodendrocyte markers. Unlike normal astrocytes, cells differentiated from glioma stem cells express both astrocytic and neuronal markers. ${ }^{90}$ Glioma stem cells also have abnormal karyotypes that are not clonal after subsequent in-vitro passaging in culture. ${ }^{74,75,91}$

\section{Transformation properties}

Glioma stem cells unlike neural stem cells exhibit anchorage independent growth under in-vitro growth conditions. Furthermore, glioma stem cells unlike neural stem cells can grow into tumours upon transplantation into immuno-deficient mice. Most remarkable, the induced tumours recapitulate the vast majority of pathological features of the parental tumour, and even retain tumour-forming potentiation on serial transplantations. ${ }^{73,75,82}$ Within the tumour mass, glioma stem cells have a bias towards forming perivascular niches ${ }^{92}$, possibly as a consequence that this micro-environment rapidly replenishes the pertinent nutrients needed to fulfill their high proliferation and differentiation indices. Secondly, glioma stem cells are highly invasive within the nervous system both locally and distal, compared to neural stem cells. In this manner, they migrate extensively along white matter tracts to distant sites in the host brain, with a

TABLE 2: Similarities and differences between a neural stem cell and glioma stem cell

\begin{tabular}{l|c|c}
\hline Sphere formation & Neural Stem Cell & Glioma Stem Cell \\
\hline Multi-potency & Yes & Tumorsphere \\
\hline Karyotype & Normal & Yes \\
\hline $\begin{array}{l}\text { Self-renewal \& } \\
\text { proliferation }\end{array}$ & Yes & Yes (enhanced) \\
\hline Clonality & High & Low \\
\hline Tumourigeneic & No & Yes \\
\hline Invasiveness & Low & High \\
\hline
\end{tabular}


tendency to destroy normal neural stem cells, a phenomenon very similar to the clinical behavior of high grade gliomas in patients. 2,93

\section{Pitfalls of glioma stem cell pre-clinical models}

The recent emergence in the use of glioma stem cells have contributed to a significant advancement in the development of novel pre-clinical models that have vast recapitulations of the pathological features of the human gliomas. ${ }^{73,75,82}$ However, the major pitfall of glioma stem cell models is the problem of de-novo molecular and pathological signatures that emerge during subsequent progressive passages during invitro culturing. ${ }^{91}$ This phenomenon is most likely a consequence of their intrinsic genetic instability that is progressively apparent upon in-vitro culturing. ${ }^{91}$ Moreover, there is a current notion that the glioma stem cells harvested from the tumour mass when cultured can become inherently different. These current observations underscore the importance of perhaps working with fresh glioma surgical samples and early passaged cells (usually $<5$ passages), to surpass these pitfalls. For instance, the tumours that arise after transplantation of established cancer cell lines ${ }^{94}$ loose their invasive properties with a tendency of not migrating along the white matter tract, compared to glioma stem cells harvested from fresh glioma surgical specimens. ${ }^{90}$ Secondly, due to inherent clonality problems, glioma stem cell models have variable and spurious therapeutic responses in the context of chemo and radio sensitivity studies. ${ }^{80-83}$

\section{Applications of stem cell based pre-clinical models to understanding treatment responses among astrocytoma patients}

Of all the stem-cell based models, glioma stem cells are the only one used so far in therapeutic response studies. Nonetheless, described below are recent findings on how current radio- and chemo- therapies affect the glioma stem cell populations within the tumour mass.

\section{Responses to radiation therapy}

Radiation therapy has been the principal means to impinge improved survival benefits in the treatments of post-operative patients, especially for those diagnosed with glioblastoma multiforme. ${ }^{95}$ However, recent preclinical studies on the glioma stem cell population have demonstrated that these cells are overwhelmingly radio-resistant to clinically relevant doses of radiation $^{80}$, and may therefore be responsible for tumour re-growth and increased aggressiveness after standard treatment measures succumbing to an eventual poor prognosis among treated patients. Specifically, radiotreatments of cells in culture exhibit that the nonglioma stem cell populations of the tumour mass are conducive to apoptosis induced cell death, in contrary to the glioma stem cell populations that remained resilient towards such treatment. In this manner, treated glioma stem cells retained their multi-lineage differentiation property, and most importantly, remained immensely tumourigeneic. What is known, thus far, with respect to the molecular mechanisms is that radioresistance in glioma stem cells is promoted via the constitutive activation of cell cycle regulatory proteins and DNA repair pathway proteins including RAD17, CHK1, CHK2 and ATM. ${ }^{80}$ The importance of these molecular findings is yet to be investigated with relevance towards novel therapeutic targeting strategies.

\section{Responses to chemotherapy drugs}

Overall, adjuvant chemotherapy has not been demonstrated to be of major clinical benefit among patients. An exception is the recent report of combined and adjuvant administration of temozolomide, which demonstrated an increase of $14 \%$ positive response in the number of glioblastoma multiforme patients who also had a median increase of 2.5 months in prolonged survival, compared to patients treated only with external radiation therapy. ${ }^{4,96}$ In general, most patients develop recurrence or progression after standard treatment measures. Glioma stem cells have elegant multi- 
drug resistance that is mediated via several mechanisms. ${ }^{81}$ Genes implicated in such multi-drug resistance include $\mathrm{BCRP} 1$. BCRP1 is a member of the ATP binding cassette (ABC) transporters comprising of 48 members. ${ }^{97}$ These transporters traffic molecules such as lipids and xenobiotic compounds across the cell membrane in an ATP dependent mechanism ${ }^{97}$. Another gene is MGMT, which is required to repair $\mathrm{O}^{6}$-methylguanine DNA induced damage by drugs such as temozolomide. If left un-repaired, this modified base can mis-pair with deoxy-thymidine during DNA replication, succumbing to acquired mutations and increased genetic instability that may potentially induce cell death. ${ }^{98}$ Anti-apoptosis pathway genes such as BCL2, FLIP, BCL-XL and IAPs (XIAP, cIAP1, cIAP2, NAIP and SURVIVIN) also confer chemo-resistance in glioma stem cells upon treatment with a plethora of chemo-therapy drugs. These genes also have an association with chemo-resistance in other cancers including acute myeloid leukemia, nonsmall cell cancer and breast cancer. ${ }^{81}$ Studies are yet to be explored which specifically address the use of combined targeted therapies, including novel drugs, that can inhibit these multi-drug resistance pathways. However, one recent and significant progress has been achieved by the identification of at least 160 new drugs that seem to effectively eradicate the glioma stem cell populations. ${ }^{99}$ The mechanisms of how these novel drugs mediate such pharmacological responses are yet to be elucidated.

\section{Concluding remarks}

Cancer associated deaths are an emerging epidemic in North America. Amongst all brain tumour cases, patients diagnosed with astrocytomas are the most common. Unfortunately despite current treatments, the majority of patients demonstrate little or no prospects for a successful prognosis. The overall impact on the healthcare system for treatments is progressively becoming more expensive. Furthermore, the psychosocial constraints on the families are devastat- ing. These treatment failures are predominantly due to the current lack of robust pre-clinical models that recapitulate the pathological, molecular and treatment characteristics of the human cancers. The recent discoveries of stem cells or immature/early-differentiated cell types as being more reminiscent in the origin of astrocytomas; and the development of stem-cell based pre-clinical models, are pre-requisites towards a fruitful venue in our quest to understand the biology of astrocytomas, and most importantly with applied prospects for more effective translational therapeutic studies. These models and applied therapeutic intervention studies will eventually assist towards a bench to bedside transition in our collective effort towards the eradication of this deadly disease among those affected.

\section{References}

1. American Cancer Society. Cancer facts and figures2001. Atlanta (GA): American Cancer Society (2001).

2. Surawicz, TS, et al. Descriptive epidemiology of primary brain and CNS tumors: results from the Central Brain Tumor Registry of the United States, 1990-1994. Neurooncology 1999 1:14-25.

3. Kleihues P, Cavenee WK. Pathology and Genetics of tumors of the nervous system: World Health Organization Classification of tumors. Oxford, Oxford Press 2000.

4. Stupp R, et al. Radiotherapy plus concomitant and adjuvant temozolomide for glioblastoma. N Engl J Med. 2005 352:987-96.

5. Rasheed BK, et al. Molecular pathogenesis of malignant gliomas. Curr Opin Oncol. 1999 11, 162-7.

6. Louis DN, Gusella JF. A tiger behind many doors: multiple genetic pathways to malignant glioma. Trends Genet. 1995;11:412-5.

7. Besson A, Yong VW. Mitogenic signaling and the relationship to cell cycle regulation in astrocytomas. $\mathrm{J}$ Neurooncol. 2001;51:245-64.

8. Huang, H., et al. Gene expression profiling of lowgrade diffuse astrocytomas by cDNA arrays. Cancer Res.2000;60:6868-74.

9. Freije, W.A., et al. Gene expression profiling of gliomas strongly predicts survival. Cancer Res. 2004;64:6503-10. 
10. Otomo, $\mathrm{T}$, et al. Microarray analysis of temporal gene responses to ionizing radiation in two glioblastoma cell lines: up-regulation of DNA repair genes. J Radiat Res (Tokyo). 2004 45, 53-60.

11. Mischel, PS, et al. Identification of molecular subtypes of glioblastoma by gene expression profiling. Oncogene. 2003;22:2361-73.

12. Qi ZY, et al. Isolation of novel differentially expressed genes related to human glioma using cDNA microarray and characterizations of two novel full-length genes. J Neurooncol. 2002;56:197-208.

13. Kotliarov Y, et al. High-resolution Global Genomic Survey of 178 Gliomas Reveals Novel Regions of Copy Number Alteration and Allelic Imbalances. Cancer Res 2006;66:9428-36.

14. Maher EA, et al. Marked genomic differences characterize primary and secondary glioblastoma subtypes and identify two distinct molecular and clinical secondary glioblastoma entities. Cancer Res. 2006;66:11502-13.

15. Dai C, et al. PDGF autocrine stimulation dedifferentiates cultured astrocytes and induces oligodendrogliomas and oligoastrocytomas from neural progenitors and astrocytes in vivo. Genes Dev. 2001;15:1913-25.

16. Sanai N, Alvarez-Buylla A, Berger MS. Neural stem cells and the origin of gliomas. N Engl J Med. 2005;353:811-22.

17. Doetsch F, et al. Subventricular zone astrocytes are neural stem cells in the adult mammalian brain. Cell. 1999;97:703-16.

18. Hopewell JW. The subependymal plate and the genesis of gliomas. J Pathol. $1975 ; 117: 101-3$.

19. Lantos PL, Cox DJ. The origin of experimental brain tumors: a sequential study. Experimentia 1976;32:1467-8.

20. Vick NA, Lin MJ, Bigner DD. The role of the subependymal plate in glial tumorigenesis. Acta Neuropathol (Berl). 1977;40:63-71.

21. Holland EC, et al. A constitutively active epidermal growth factor receptor cooperates with disruption of G1 cell-cycle arrest pathways to induce glioma-like lesions in mice. Genes Dev. 1998 12:3675-85.

22. Holland EC, et al. Combined activation of Ras and Akt in neural progenitors induces glioblastoma formation in mice. Nat Genet. 2000 25:55-7.

23. Uhrbom L, et al. Ink4a-Arf loss cooperates with KRas activation in astrocytes and neural progenitors to generate glioblastomas of various morphologies depending on activated Akt. Cancer Res. 2002;62:5551-8.

24. Marumoto T, et al. Development of a novel mouse glioma model using lentiviral vectors. Nat Med. 2009;15:110-6.
25. Bewley MC, et al. Structural analysis of the mechanism of adenovirus binding to its human cellular receptor, CAR. Science. 1999;286(5444):1579-83.

26. Zhu Y, et al. Early inactivation of p53 tumor suppressor gene cooperating with NF1 loss induces malignant astrocytoma. Cancer Cell. 2005;8:119-30.

27. Sonoda Y, et al. Formation of intracranial tumors by genetically modified human astrocytes defines four pathways critical in the development of human anaplastic astrocytoma. Cancer Res. 2001a:61:4956-60.

28. Sonoda Y, et al. Akt pathway activation converts anaplastic astrocytoma to glioblastoma multiforme in a human astrocyte model of glioma. Cancer Res. 2001b;61:6674-8.

29. Kamnasaran D, et al. GATA6 is an astrocytoma tumor suppressor gene identified by gene trapping of mouse glioma model. Proc Natl Acad Sci U S A. 2007;104:8053-8.

30. Biernaskie JA, et al. Isolation of skin-derived precursors (SKPs) and differentiation and enrichment of their Schwann cell progeny. Nat Protoc. 2006;1:2803-12.

31. Biernaskie J, et al. Skin-derived precursors generate myelinating Schwann cells that promote remyelination and functional recovery after contusion spinal cord injury. J Neurosci. 2007;27:9545-59.

32. Bachoo RM, et al. Epidermal growth factor receptor and Ink4a/Arf: convergent mechanisms governing terminal differentiation and transformation along the neural stem cell to astrocyte axis. Cancer Cell. 2002;1:269-77.

33. Kamnasaran D, Hawkins C, Guha A. Characterization and transformation potential of "Synthetic" astrocytes differentiated from murine embryonic stem cells. Glia. 2008;56:457-70.

34. Jang YK, et al. Retinoic acid-mediated induction of neurons and glial cells from human umbilical cordderived hematopoietic stem cells. J Neurosci Res. 2004;75:573-84.

35. Lee MW, et al. Neural differentiation of novel multipotent progenitor cells from cryopreserved human umbilical cord blood. Biochem Biophys Res Commun. 2007;358:637-43.

36. Mokrý J, Karbanova J, Filip S. Differentiation potential of murine neural stem cells in vitro and after transplantation. Transplant Proc. 2005 ;37:268-72.

37. Rao MS, Noble M, Mayer-Pröschel M. A tripotential glial precursor cell is present in the developing spinal cord. Proc Natl Acad Sci U S A. 1998;95:3996-4001.

38. Yang SY, Liu H, Zhang JN. Gene therapy of rat malignant gliomas using neural stem cells expressing IL-12. DNA Cell Biol. 2004;23:381-9. 
39. Tang F, et al. Differentiation of embryonic stem cell to astrocytes visualized by green fluorescent protein. Cell Mol Neurobiol. 2002;22:95-101.

40. Mi H, Haeberle H, Barres BA. Induction of astrocyte differentiation by endothelial cells. J Neurosci. 2001;21:1538-47.

41. Chang MY, et al. Neurons and astrocytes secrete factors that cause stem cells to differentiate into neurons and astrocytes, respectively. Mol Cell Neurosci. 2003;23:414-26.

42. Jori FP, et al. EGF-responsive rat neural stem cells: molecular follow-up of neuron and astrocyte differentiation in vitro. J Cell Physiol. 2003;195:220-33.

43. Rajan P, McKay RD. Multiple routes to astrocytic differentiation in the CNS. J Neurosci. 1998;18:3620-9.

44. Satoh M, Sugino H, Yoshida T. Activin promotes astrocytic differentiation of a multipotent neural stem cell line and an astrocyte progenitor cell line from murine central nervous system. Neurosci Lett. 2000;284:143-6.

45. Hajihosseini MK, Dickson C. A subset of fibroblast growth factors (Fgfs) promote survival, but Fgf- 8 b specifically promotes astroglial differentiation of rat cortical precursor cells. Mol Cell Neurosci. 1999;14:468-85.

46. Mabie PC, et al. Bone morphogenetic proteins induce astroglial differentiation of oligodendroglial-astroglial progenitor cells. J Neurosci. 1997;17:4112-20.

47. Reynolds BA, Weiss S. Generation of neurons and astrocytes from isolated cells of the adult mammalian central nervous system. Science. 1992;255:1707-10.

48. Kilpatrick TJ, Bartlett PF. Cloning and growth of multipotential neural precursors: requirements for proliferation and differentiation. Neuron. 1993;10:255-65.

49. Temple S. The development of neural stem cells. Nature. $2001 ; 414: 112-7$.

50. Colombo E, et al. Embryonic stem-derived versus somatic neural stem cells: a comparative analysis of their developmental potential and molecular phenotype. Stem Cells. 2006;24:825-34.

51. Lim JK, et al. Brain-derived neurotrophic factor stimulates the neural differentiation of human umbilical cord blood-derived mesenchymal stem cells and survival of differentiated cells through MAPK/ERK and PI3K/ Akt-dependent signaling pathways. J Neurosci Res. 2008;86:2168-78.

52. Evans MJ, Kaufman MH. Establishment in culture of pluripotential cells from mouse embryos. Nature. 1981;292:154-6.

53. Martin GR. Isolation of a pluripotent cell line from early mouse embryos cultured in medium conditioned by teratocarcinoma stem cells. Proc Natl Acad Sci U S
A. $1981 ; 78: 7634-8$.

54. Thomson JA, et al. Isolation of a primate embryonic stem cell line. Proc Natl Acad Sci U S A. 1995;92:7844-8.

55. Thomson JA, et al. Pluripotent cell lines derived from common marmoset (Callithrix jacchus) blastocysts. Biol Reprod. 1996;55:254-9.

56. Thomson JA, et al. Embryonic stem cell lines derived from human blastocysts. Science. 1998;282:1145-7.

57. Suemori H, et al. Establishment of embryonic stem cell lines from cynomolgus monkey blastocysts produced by IVF or ICSI. Dev Dyn. 2001;222:273-9.

58. Reubinoff BE, et al. Embryonic stem cell lines from human blastocysts: somatic differentiation in vitro. Nat Biotechnol. 2000;18:399-404.

59. Brustle O, et al.. Embryonic stem cell-derived glial precursors: a source of myelinating transplants. Science. 1999;285:754-6.

60. Fraichard A, et al. In vitro differentiation of embryonic stem cells into glial cells and functional neurons. J Cell Sci. 1995;108:3181-8.

61. Slager HG, et al. Transforming growth factor-beta in the early mouse embryo: implications for the regulation of muscle formation and implantation. Dev Genet. 1993;14:212-24.

62. Palmer TD, et al. Cell culture. Progenitor cells from human brain after death. Nature 2001;411:42-3.

63. Gomes WA, Mehler MF, Kessler JA. Transgenic overexpression of BMP4 increases astroglial and decreases oligodendroglial lineage commitment. Dev Biol. 2003;255:164-77.

64. Nakashima K, et al. Astrocyte differentiation mediated by LIF in cooperation with BMP2. FEBS Lett. 1999;457:43-6.

65. Fan Y, Melhem MF, Chaillet JR. Forced expression of the homeobox-containing gene Pem blocks differentiation of embryonic stem cells. Dev Biol. 1999;210:481-96.

66. Groszer M, et al. Negative regulation of neural stem/ progenitor cell proliferation by the Pten tumor suppressor gene in vivo. Science. 2001;294:2186-9.

67. Kielman MF, et al. Apc modulates embryonic stem-cell differentiation by controlling the dosage of betacatenin signaling. Nat Genet. 2002;32:594-605.

68. Penninger JM, Woodgett J. Stem cells. PTEN-coupling tumor suppression to stem cells? Science. $2001 ; 294: 2116-8$.

69. Field SJ, et al. Growth and differentiation of embryonic stem cells that lack an intact c-fos gene. Proc Natl Acad Sci U S A. 1992;89:9306-10. 
70. Clarke MF, et al. Cancer stem cells--perspectives on current status and future directions: AACR Workshop on cancer stem cells. Cancer Res. 2006;66:9339-44.

71. Ignatova TN, et al. Human cortical glial tumors contain neural stem-like cells expressing astroglial and neuronal markers in vitro. Glia. 2002 ;39:193-206.

72. Hemmati HD, et al. Cancerous stem cells can arise from pediatric brain tumors. Proc Natl Acad Sci U S A. 2003;100:15178-83.

73. Galli R, et al. Isolation and characterization of tumorigenic, stem-like neural precursors from human glioblastoma. Cancer Res. 2004;64:7011-21.

74. Singh SK, et al. Identification of a cancer stem cell in human brain tumors. Cancer Res. 2003;63:5821-8.

75. Singh SK, et al. Identification of a cancer stem cell in human brain tumors. Cancer Res. 2003;63:5821-8.

76. Yuan $X$, et al. Isolation of cancer stem cells from adult glioblastoma multiforme. Oncogene. 2004;23:9392-400.

77. Rebetz J, et al. Glial progenitor-like phenotype in lowgrade glioma and enhanced CD133-expression and neuronal lineage differentiation potential in high-grade glioma. PLoS ONE. 2008;3:e1936.

78. Kondo T, Setoguchi T, Taga T. Persistence of a small subpopulation of cancer stem-like cells in the C6 glioma cell line. Proc Natl Acad Sci U S A. 2004;101:781-6.

79. $\mathrm{Yu}$ SC, et al. Isolation and characterization of cancer stem cells from a human glioblastoma cell line U87. Cancer Lett. 2008;265:124-34.

80. Bao S, et al., Glioma stem cells promote radioresistance by preferential activation of the DNA damage response. Nature. 2006;444:756-60.

81. Liu G, et al. Analysis of gene expression and chemoresistance of CD133+ cancer stem cells in glioblastoma. Mol Cancer. 2006;5:67.

82. Salmaggie A, et al. Glioblastoma-derived tumorospheres identify a population of tumor stem-like cells with angiogenic potential and enhanced multidrug resistance phenotype. Glia. 2006;54:850-60.

83. Kang MK, Kang SK. Tumorigenesis of chemotherapeutic drug-resistant cancer stem-like cells in brain glioma. Stem Cells Dev. 2007;16:837-47.

84. Sanai N, et al. Unique astrocyte ribbon in adult human brain contains neural stem cells but lacks chain migration. Nature. 200; 427:740-4.

85 . Doetsch F. The glial identity of neural stem cells. Nat Neurosci. 2003;6:1127-34.

86. Jackson EL, et al. PDGFR alpha-positive B cells are neural stem cells in the adult SVZ that form gliomalike growths in response to increased PDGF signaling. Neuron. 2006;51:187-99.
87. Beier D, et al. CD133(+) and CD133(-) glioblastomaderived cancer stem cells show differential growth characteristics and molecular profiles. Cancer Res. 2007;67:4010-5.

88. Carpenter MK, et al. Properties of four human embryonic stem cell lines maintained in a feeder-free culture system. Dev Dyn. 2004;229:243-58.

89. Serakinci N, Graakjaer J, Kolvraa S. Telomere stability and telomerase in mesenchymal stem cells. Biochimie. 2008;90:33-40.

90. Varghese M, et al. A comparison between stem cells from the adult human brain and from brain tumors. Neurosurgery. 2008;63:1022-33.

91. Tunici $P$, et al. Genetic alterations and in vivo tumorigenicity of neurospheres derived from an adult glioblastoma. Mol Cancer. $2004 ; 3: 25$.

92. Calabrese $\mathrm{C}$, et al. A perivascular niche for brain tumor stem cells. Cancer Cell. 2007;11:69-82.

93. Lefranc F, et al. Present and potential future issues in glioblastoma treatment. Expert Rev Anticancer Ther. 2006;6:719-32.

94. Lee J, Kotliarova S, Kotliarov Y, et al. Tumor stem cells derived from glioblastomas cultured in bFGF and EGF more closely mirror the phenotype and genotype of primary tumors than do serum-cultured cell lines. Cancer Cell. 2006;9:391-403.

95. Walker MD, et al. Randomized comparisons of radiotherapy and nitrosoureas for the treatment of malignant glioma after surgery. N Engl J Med. 1980;303:1323-9.

96. Taphoorn MJ et al. Health-related quality of life in patients with glioblastoma: a randomised controlled trial. Lancet Oncol. 2005;6:937-44.

97. Lu C, Shervington A. Chemoresistance in gliomas. Mol Cell Biochem. 2008 ;312:71-80.

98. Denny BJ, et al. NMR and molecular modeling investigation of the mechanism of activation of the antitumor drug temozolomide and its interaction with DNA. Biochemistry. 1994;33:9045-51.

99. Diamandis P, et al. Chemical genetics reveals a complex functional ground state of neural stem cells. Nat Chem Biol. 2007;3:268-73

\section{Correspondence to:}

Dr. Deepak Kamnasaran

Pediatrics Research Unit

Centre de Recherche du CHUL (CHUQ)

2705, boulevard Laurier, Local RC-9800

Québec, Québec, CANADA

G1V 4G2

E-mail: deepak.kamnasaran@crchul.ulaval.ca 